Bemerkt sei, daß der Alkoholgehalt der Ammoniumpersulfatlösungen allmählich zu Aldehyd oxydiert wird, was aber den Gebrauch zur Erzeugung der Jodtinktur nicht stört. Nur sehr alte Lösungen, die einige Wochen gestanden haben, sind nicht mehr so wirksam.

Wenn ich in therapeutischer Hinsicht die beiden Methoden zur Erzeugung von Jodtinktur in statiz nascendi vergleiche, so kommen sie beide zur Desinfektion des Operationsfeldes gebraucht werden. Zur therapeutischen Verwendung beispielsweise bei parasitären Prozessen der Haut, wo uns außcr der bakteriziden Wirkung noch eine desquamicrende erwïnscht ist, ist die Erzeugung durch Jodicum der in dieser Arbeit angegebenen Methode vorzuziehen.

Literatur 1 M. m. W $1913 \mathrm{Nr}, 47-2 \mathrm{D}, \mathrm{m} \mathrm{W} 1914 \mathrm{Nr} 22$

\title{
Zur Desinfektion des Operationsfeldes mit Jodtinktur oder anderen Arzneimitteln.
}

Von J. Schumacher in Berlin.

Die Frage hat zweifelsohne in erster Linie für den Feldarzt Interesse, wenn beispielsweise der vorhandene Vorrat an Jodtinktur ver braucht ist, aber Ersatz nicht nachgeschafft oder auf irgendeine Weise vernichtet wurde.

Daß man aus bestimmten Gründen zweckmäßig mit stets f rischer Jodtinktur arbeitet, darauf haben Bachem (1) und ich (2) bereits hingewiesen und die Gründe dafür auseinandergesetzt. Dort habe ich auch auf die Vorteile hingewiesen, die sich bieten, wenn man den Jodanstrich auf der Haut selbst erst chemisch aus zwei verschiedenen Lösungen in statu nascendi entstehen läßt.

Daß man sich der Jodicumtabletten bedienen kann, die die Jodtinktur in fester Form bequem nachführen lassen, bei geringer Rauminanspruchnahme, das habe ich schon betont. Mit Hilfe von Alkohol und Kornbranntwein kann sich auf diese Weise der Landarzt Geburtshelfer oder Feldarzt jederzeit sofort eine frische Jodtinktur be reiten. Kornbranntwein befindet sich wohl in jedem Dorf, auch Brennspiritus ist dazu geeignet, soll jedoch nicht dazu verwendet werden seines Methylalkoholgehaltes wegen, wenn meiner Ansicht nach auch kein Grund dafur vorliegt. daB bei der geringen Menge Flussigkeit, die zu einem Jodanstrich nötig ist, nennenswerte Mengen resorbiert werden konnen, zumal durch die intaktc Haut.

Doch hierbei ist ebenfalls Voraussetzung, daß man den Jodtinkturersatz zur Hand hat.

Ich war daher bemuht, bei nicht vorhandencr Jodtinktur einen Ersatz zu schaffen unter Zuhilfenahme von Medikamenten, die zu unserem therapeutischen Schatz gehören. Ich verwende dazu dic alkoholischen Lösungen von Jodkalium und Ammoniumpersulfat dem von mir eingeführten Antigonorrhoicum. Man stellt sich zweckmaßig folgende zwei Lösungen ber oder hält sie sich vorrätig:

$$
\text { L. } \mathbf{R p} \text {. }
$$

Kal. jodati 2,0

Aquae

Spiritus aa 5,0

II. $R_{p}$

Ammoniumpersulfat 2,0

Aquae

Spiritus aa 5,

Man taucht ein mit einem Wattebausch armiertes Stäbchen in die erste Lösung und bestreicht damit die Haut. Mit einem anderen, in die zweite Lösung getauchten bestreicht man alsdann dic vorher behandelte Flache. Der Jodanstrich, der sofort entsteht, ist in den ersten zwei Sekunden noch hellgelb, sobald jedoch die Umsetzung zu Ende ist, was nack funfzig Sekunden der Fall ist, ist der Anstrich von dem mit Jodtinktur gemachten nicht mehr zu unterscheiden. Ein Mischen der beiden Flïssigkeiten empfiehlt sich nicht, da hicrbei cin alkoholunlóslicher Nicderschlag entsteht, was aber weiter den Gebrauch der betreffenden Flïssigkeiten zur Jodierung der Haut nicht stört. Yur dauert hierbei die Entstehung der Jodtinktur längere Zeit, im Gegensatz zum Gebrauch von Jodicum, wo diese beim Vermischen momentan entsteht. 